\title{
Characterization of uniform statistical convergence for double sequences
}

\author{
E. Tas and T. Yurdakadim
}




\title{
CHARACTERIZATION OF UNIFORM STATISTICAL CONVERGENCE FOR DOUBLE SEQUENCES
}

\author{
E. TAS AND T. YURDAKADMIN
}

Received 30 March, 2011

\begin{abstract}
In the present study we introduce uniform statistical convergence for double sequences. We present a decomposition theorem that characterizes uniform statistical convergence for double sequences.
\end{abstract}

2000 Mathematics Subject Classification: 40B05; 40G15

Keywords: double sequences, uniform density, uniform statistical convergence, almost convergence

\section{INTRODUCTION}

In the summability theory uniform statistical convergence has important roles for ordinary (single) sequences. Since this type of convergence method is stronger than ordinary convergence, it is quite effective, especially when the classical limit does not exist. This idea has been introduced by Brown and Freedman [2] by using the notion of uniform density. Then the method has been discussed by many authors in various directions ([1], [4] and [5]). The concept of uniform density has recently been extended to double sequences [14].

In the present work we introduce the uniform statistical convergence by using the uniform density for double sequences. We present a decomposition theorem that characterizes uniform statistical convergence for double sequences and show that for double sequences almost convergence and statistical convergence are not compatible. Also we give a relation between uniform statistical convergence and strong uniform p-Cesàro summable double sequences. Finally by defining strongly almost convergence with respect to a modulus function we examine the new sequence space together with the space of all uniformly statistically convergent sequences.

In 1900 Pringsheim [13] introduced the concept of convergence for double sequences. A double sequence $x=\left(x_{i j}\right)$ is said to be convergent in Pringsheim sense if for every $\varepsilon>0$ there exists an $N \in \mathbb{N}$ such that $\left|x_{i j}-L\right|<\varepsilon$ whenever $i, j \geq N$. In this case $L$ is called the Pringsheim limit of $x$ and space of such sequences is denoted by $c_{2}$. A double sequence $x$ is bounded if there exists a positive number $M$ such that 
$\left|x_{i j}\right|<M$ for all $i$ and $j$,i.e.,

$$
\|x\|_{(\infty, 2)}=\sup _{i, j}\left|x_{i j}\right|<\infty .
$$

We will denote the set of all bounded double sequences by $l_{\infty}^{2}$. Note that in contrast to the case for single sequences, a convergent double sequence need not be bounded.

Let $K \subseteq \mathbb{N} \times \mathbb{N}$ be a two dimensional set of positive integers and let $K(n, m)$ be the numbers of $(i, j)$ in $K$ such that $i \leq n, j \leq m$. Then the lower asymptotic density of a set $K \subseteq \mathbb{N} \times \mathbb{N}$ is defined as

$$
\underline{\mathrm{d}}_{2}(K)=\liminf _{n, m} \frac{K(n, m)}{n m}
$$

and the upper asymptotic density is defined as

$$
\bar{d}_{2}(K)=\limsup _{n, m} \frac{K(n, m)}{n m} .
$$

If $\bar{d}_{2}(K)=\underline{\mathrm{d}}_{2}(K)$ then $d_{2}(K)=\bar{d}_{2}(K)=\underline{\mathrm{d}}_{2}(K)$ is called the asymptotic density [11].

The following definition has been given in [10], [11] and [15] independently.

Definition 1. A double sequence $x=\left(x_{i j}\right)$ is said to be statistically convergent if for every $\varepsilon>0, d_{2}\left(A_{\varepsilon}\right)=0$ where $A_{\varepsilon}=\left\{(i, j) \in \mathbb{N} \times \mathbb{N}:\left|x_{i j}-L\right| \geq \varepsilon\right\}$.

We will denote the set of all statistically convergent double sequences by $s t^{2}$. The lower uniform density of a set $K \subseteq \mathbb{N} \times \mathbb{N}$ is defined as

$$
\underline{\mathrm{u}}_{2}(K)=\lim _{p, q} \frac{\min _{m, n \geq 0}|\{(i, j) \epsilon K: m+1 \leq i \leq m+p, n+1 \leq j \leq n+q\}|}{p q}
$$

and the upper uniform density is defined as

$$
\bar{u}_{2}(K)=\lim _{p, q} \frac{\max _{m, n \geq 0}|\{(i, j) \epsilon K: m+1 \leq i \leq m+p, n+1 \leq j \leq n+q\}|}{p q} .
$$

If $\bar{u}_{2}(K)=\underline{\mathrm{u}}_{2}(K)$ then $u_{2}(K)=\bar{u}_{2}(K)=\underline{\underline{u}}_{2}(K)$ is called uniform density.

It is clear that $\underline{\mathrm{u}}_{2}(K) \leq \underline{\mathrm{d}}_{2}(K) \leq \bar{d}_{2}(K) \leq \bar{u}_{2}(K)$.

We now introduce the concept of uniform statistical convergence for double sequences.

Definition 2. A double sequence $x=\left(x_{i j}\right)$ is said to be uniformly statistically convergent if for every $\varepsilon>0, u_{2}\left(A_{\varepsilon}\right)=0$ where $A_{\varepsilon}=\left\{(i, j) \in \mathbb{N} \times \mathbb{N}:\left|x_{i j}-L\right| \geq \varepsilon\right\}$.

By $s t_{u}^{2}$ we will denote the set of all statistically convergent double sequences. 

$l_{\infty}^{2}$

Note that the set $s t_{u}^{2} \cap l_{\infty}^{2}$ is a closed linear subspace of the normed linear space

Uniform statistical convergence is closely related to statistical convergence. As in the case of single sequences, we have the following inclusions

$$
c_{2} \subset s t_{u}^{2} \subset s t^{2} .
$$

These inclusions are proper as the following examples show.

Example 1. Let $P$ be the set of all primes. Define a double sequence $x=\left(x_{i j}\right)$ as follows

$$
x_{i j}= \begin{cases}1 & ; \quad(i, j) \in P \times P \\ 0 & ; \text { otherwise. }\end{cases}
$$

We know that $u(P)=0$ [3]. It is easy to see that $u_{2}(P \times P)=0$. Hence $x=\left(x_{i j}\right)$ is uniformly statistically convergent to 0 but not convergent.

Example 2. Let $A=\bigcup_{k=1}^{\infty}\left\{5^{k}+1,5^{k}+2, \ldots, 5^{k}+k\right\}$. Define a double sequence $x=\left(x_{i j}\right)$ as follows

$$
x_{i j}=\left\{\begin{array}{cc}
1 & ; \quad(i, j) \in A \times A \\
0 & ; \quad \text { otherwise. }
\end{array}\right.
$$

It is known from [1] that $\underline{\mathrm{u}}(A)=0, \bar{u}(A)=1, d(A)=0$.

One can easily see that $\underline{\underline{u}}_{2}(A \times A)=0, \bar{u}_{2}(A \times A)=1, d_{2}(A \times A)=0$. Hence the double sequence $x$ is statistically convergent to 0 , but not uniformly statistically convergent.

\section{CHARACTERIZATIONS OF UNIFORM STATISTICAL CONVERGENCE}

This section presents some comparison results which are related to uniform statistical convergence for double sequences. Also uniform statistical convergence is characterized.

The next definition may be found in [8] and [9].

Definition 3. A double sequence $x=\left(x_{i j}\right)$ is called almost convergent to $L$ if

$$
\lim _{p, q} \frac{1}{p q} \sum_{i, j=m+1, n+1}^{m+p, n+q} x_{i j}=L \text {, (uniformly in } n \text { and } m \text { ). }
$$

By $a c^{2}$, we denote the set of almost convergent double sequences.

Recall that $a c^{2} \subset l_{\infty}^{2},[8]$.

We know that almost convergence and statistical convergence are not compatible for ordinary sequences [7]. This raises the question of whether this property holds for double sequences. The following examples demonstrates this fact in the affirmative. 
Example 3. Let $x_{i j}=\left\{\begin{array}{cc}i j & ; \quad i \text { and } j \text { square } \\ 0 & ; \quad \text { otherwise }\end{array}\right.$.

Since $x$ is unbounded, it can not be almost convergent. But it is easy to see that $x$ is statistically convergent to 0 .

Example 4. Let $x_{i j}=\left\{\begin{array}{ccc}0 & ; & i \text { is even, for every } j \\ 1 & ; & i \text { is odd, for every } j\end{array}\right.$.

One can observe that $x$ can not be statistically convergent but it is almost convergent to $\frac{1}{2}$.

For double sequences almost convergence was introduced by Mòricz and Rhoades [8] and it was studied in some detail in [16] and [9].

The following definition is an extension of a definition given in [1] to double sequences.

Definition 4. A double sequence $x=\left(x_{i j}\right)$ is said to be uniformly strong $p$-Cesàro convergent $(0<p<\infty)$ to $L$ if

$$
\lim _{k, l} \frac{1}{k l} \sum_{i, j=m+1, n+1}^{m+k, n+l}\left|x_{i j}-L\right|^{p}=0 \text {, (uniformly in } n \text { and } m \text { ). }
$$

By $u w_{p}^{2}$, we denote the set of all uniformly strongly $p$-Cesàro convergent double sequences. It is immediate that $u w_{p}^{2} \subset w_{p}^{2}$, where $w_{p}^{2}$ denotes space of all strongly $p$-Cesàro convergent double sequences studied in [11].

In case $p=1, x$ is said to be strongly almost convergent. So this implies almost convergence.

It is known that $c \subset a c$ for single (ordinary) sequences. But we have $l_{\infty}^{2} \cap c_{2} \subset a c^{2}$ for double sequences ([8]).

We now give our first characterization for uniform statistical convergence of double sequences.

Theorem 1. (i) If $0<p<\infty$ and a sequence $x=\left(x_{i j}\right)$ is uniformly strongly $p$-Cesàro convergent to $L$, then it is uniformly statistically convergent to $L$.

(ii) If $x=\left(x_{i j}\right)$ is bounded and uniformly statistically convergent to $L$, then it is uniformly strongly $p$-Cesàro convergent to L for every $p, 0<p<\infty$.

Proof. (i) Let $x$ be uniformly strongly $p$-Cesàro convergent to $L, 0<p<\infty$. Suppose $\varepsilon>0$. Then for every $n, m \in \mathbb{N}$, we have

$$
\sum_{i, j=1,1}^{k, l}\left|x_{n+i, m+j}-L\right|^{p} \geq \sum_{\substack{i, j=1,1 \\\left|x_{n+i, m+j}-L\right| \geq \varepsilon}}^{k, l}\left|x_{n+i, m+j}-L\right|^{p}
$$




$$
\geq \varepsilon^{p} \sum_{\substack{i, j=1,1 \\\left|x_{n+i, m+j}-L\right| \geq \varepsilon}}^{k, l} 1 \geq \varepsilon^{p} A_{\varepsilon}(n+1, n+k ; m+1, m+l) .
$$

Then it follows that

$$
\frac{1}{k l} \sup _{n, m} \sum_{i, j=1,1}^{k, l}\left|x_{n+i, m+j}-L\right|^{p} \geq \varepsilon^{p} \frac{1}{k l} \sup _{n, m} A_{\varepsilon}(n+1, n+k ; m+1, m+l),
$$

where

$$
\begin{gathered}
A_{\varepsilon}(n+1, n+k ; m+1, m+l)= \\
\left\{(i, j): n+1 \leq i \leq n+k, m+1 \leq j \leq m+l ;\left|x_{i j}-L\right| \geq \varepsilon\right\} .
\end{gathered}
$$

This implies $\bar{u}_{2}\left(A_{\varepsilon}\right)=0$ and $u_{2}\left(A_{\varepsilon}\right)=0$, so that $s t_{u}^{2}-\lim x=L$.

(ii) Suppose that $x$ is a bounded sequence and $s t_{u}^{2}-\lim x=L$. Let $0<p<\infty$ and $\varepsilon>0$. According to the hypothesis we have $u_{2}\left(A_{\varepsilon}\right)=0$.

The boundedness of $x=\left(x_{i j}\right)$ implies that there exists $M>0$ such that for every $i, j=1,2, \ldots,\left|x_{i j}-L\right| \leq M$.

Observe that for every $n, m \in \mathbb{N}$, we have

$$
\begin{aligned}
& \frac{1}{k l} \sum_{i, j=1,1}^{k, l}\left|x_{n+i, m+j}-L\right|^{p}= \\
& \frac{1}{k l} \sum_{\substack{i, j=1,1 \\
(n+i, m+j) \in A_{\varepsilon}}}^{k, l}\left|x_{n+i, m+j}-L\right|^{p}+\frac{1}{k l} \sum_{\begin{array}{c}
i, j=1,1 \\
(n+i, m+j) \notin A_{\varepsilon}
\end{array}}^{k, l}\left|x_{n+i, m+j}-L\right|^{p} \\
& \leq M^{p} \frac{1}{k l} \max _{n, m \geq 0} A_{\varepsilon}(n+1, n+k ; m+1, m+l)+\varepsilon^{p} \frac{1}{k l} \sum_{i, j=1,1}^{k, l} 1 \\
& \leq M^{p} u_{2}\left(A_{\varepsilon}\right)+\varepsilon^{p} .
\end{aligned}
$$

This implies that $\lim _{k, l} \frac{1}{k l} \sum_{i, j=m+1, n+1}^{m+k, n+l}\left|x_{i j}-L\right|^{p}=0$ (uniformly in $n$ and $m$ ).

We immediately obtain the following results from Theorem 1.

Corollary 1. Let $x=\left(x_{i j}\right)$ is a bounded double sequence. If $x$ is uniformly statistically convergent to $L$ then $x$ is almost convergent to $L$.

But the converse of this corollary does not hold, see Example 4.

Corollary 2. Let $x=\left(x_{i j}\right)$ is a bounded double sequence. Then $x$ is uniformly statistically convergent to $L$ if and only if $x$ is uniformly strongly $p$-Cesàro convergent to $L$ for every $p, 0<p<\infty$.

The following result characterizes uniform statistical convergence. 
Theorem 2. The following are equivalent.

(i) The double sequence $x=\left(x_{k l}\right)$ is uniformly statistically convergent to $L$.

(ii) There exists a sequence $y=\left(y_{k l}\right)$ which is convergent and such that $u_{2}\left(\left\{(k, l): x_{k l}=y_{k l}\right\}\right)=1$.

(iii) There exists a subset $M=\left\{\left(k_{i}, l_{j}\right): i, j \in \mathbb{N}\right\}$ such that $u_{2}(M)=1$ and $\left(x_{k_{i} l_{j}}\right)$ is convergent.

(iv) There exists two sequences $\left(y_{k l}\right)$ and $\left(z_{k l}\right)$ such that $x_{k l}=y_{k l}+z_{k l}$ for all $k, l$ and $\left(y_{k l}\right)$ converges to $L$ and $\left(z_{k l}\right)$ is uniformly statistically convergent to 0 .

Proof. (i) $\Rightarrow$ (ii): Let the double sequence $\left(x_{k l}\right)$ be uniformly statistically convergent to $L$. Let the complement of the set $A_{\frac{1}{j}}=\left\{(p, q):\left|x_{k_{p}} l_{q}-L\right| \geq \frac{1}{j}\right\}, j=$ $1,2, \ldots$, be

$$
K_{j}=\mathbb{N} \times \mathbb{N}-A_{\frac{1}{j}} .
$$

Hence by the definition of uniform statistical convergence, we have $u_{2}\left(K_{j}\right)=1$, for $j=1,2, \ldots$

By the definition of $K_{j}$ we have $K_{1} \supseteq K_{2} \supseteq \ldots \supseteq K_{j} \supseteq K_{j+1} \supseteq \ldots$... Let us choose an arbitrary $s_{1}=\left(s_{1}^{\prime}, s_{1}^{\prime \prime}\right) \in K_{1}$. By the definition of $K_{j}$, there exists $s_{2}=\left(s_{2}^{\prime}, s_{2}^{\prime \prime}\right) \in K_{2}$ such that $s_{2}^{\prime}>s_{1}^{\prime}, s_{2}^{\prime \prime}>s_{1}^{\prime \prime}$ and for every $p \geq s_{2}^{\prime}, q \geq s_{2}^{\prime \prime}$

$$
\frac{\min _{m, n \geq 0} K_{2}(m+1, m+p ; n+1, n+q)}{p q}>\frac{1}{2} .
$$

Again by the definition of $K_{j}$ there exists $s_{3}=\left(s_{3}^{\prime}, s_{3}^{\prime \prime}\right) \in K_{3}$ such that $s_{3}^{\prime}>s_{2}^{\prime}$, $s_{3}^{\prime \prime}>s_{2}^{\prime \prime}$ and for every $p \geq s_{3}^{\prime}, q \geq s_{3}^{\prime \prime}$.

$$
\frac{\min _{m, n \geq 0} K_{3}(m+1, m+p ; n+1, n+q)}{p q}>\frac{2}{3} .
$$

Continuing in this way, we can construct increasing sequences of positive integers $s_{1}^{\prime}<s_{2}^{\prime}<s_{3}^{\prime}<\ldots<s_{j}^{\prime}<\ldots$

$s_{1}^{\prime \prime}<s_{2}^{\prime \prime}<s_{3}^{\prime \prime}<\ldots<s_{j}^{\prime \prime}<\ldots$ such that $s_{j}=\left(s_{j}^{\prime}, s_{j}^{\prime \prime}\right) \in K_{j}$ and for every $p \geq s_{j}^{\prime}$, $q \geq s_{j}^{\prime \prime}$

$$
\frac{\min _{m, n \geq 0} K_{j}(m+1, m+p ; n+1, n+q)}{p q}>1-\frac{1}{j}, \quad \text { for } j=1,2,3, \ldots .
$$

Define $K$ as follows: if $1 \leq k \leq s_{1}^{\prime}$ or $1 \leq l \leq s_{1}^{\prime \prime}$ then $(k, l) \in K$; suppose that $j \geq 1$ and $s_{j}^{\prime}<k \leq s_{j+1}^{\prime}$ or $s_{j}^{\prime \prime}<l \leq s_{j+1}^{\prime \prime}$ then $(k, l) \in K$ if and only if $(k, l) \epsilon K_{j}$. 
For every $p, q$, such that $s_{j}^{\prime}<p \leq s_{j+1}^{\prime}$ and $s_{j}^{\prime \prime}<q \leq s_{j+1}^{\prime \prime}$, we have

$$
\begin{aligned}
\frac{\min _{m, n \geq 0} K(m+1, m+p ; n+1, n+q)}{p q} & \geq \frac{\min _{m, n \geq 0} K_{j}(m+1, m+p ; n+1, n+q)}{p q} \\
& \geq 1-\frac{1}{j} .
\end{aligned}
$$

Hence it is obvious that $u_{2}(K)=1$. Let $\varepsilon>0$ be given and select $j$ such that $\frac{1}{j}<\varepsilon$. Let $p \geq s_{j}^{\prime}, q \geq s_{j}^{\prime \prime},(p, q) \in K$. Then there exists a number $r \geq j$ such that $s_{r}^{\prime}<p \leq s_{r+1}^{\prime}$ and $s_{r}^{\prime \prime}<q \leq s_{r+1}^{\prime \prime}$. According to the definition of $K,(p, q) \in K_{r}$ is obtained, we have $\left|x_{p q}-L\right|<\frac{1}{r} \leq \frac{1}{j}<\varepsilon$. Thus $\left|x_{p q}-L\right|<\varepsilon$ for every $p \geq s_{j}^{\prime}$, $q \geq s_{j}^{\prime \prime},(p, q) \in K$. Hence $\lim _{\substack{p, q \\(p, q) \in K}} x_{p q}=L$.

Let us define $y=\left(y_{p q}\right)$ as follows

$$
y_{p q}=\left\{\begin{array}{ccc}
x_{p q} & ; \quad(p, q) \in K \\
L & ; & \text { otherwise }
\end{array} .\right.
$$

So $\lim _{p, q} y_{p q}=L$ and $u_{2}\left(\left\{(p, q): x_{p q}=y_{p q}\right\}\right)=u_{2}(\{(p, q) \epsilon K\})=1$

(ii) $\Rightarrow$ (iii): Let there exists a sequence $y=\left(y_{k l}\right)$ which is convergent such that $u_{2}\left(\left\{(k, l): x_{k l}=y_{k l}\right\}\right)=1$. Let $M=\left\{(k, l): x_{k l}=y_{k l}\right\}$ then $u_{2}(M)=1$. Now we can represent $M$ as $M=\left\{\left(k_{i}, l_{j}\right): i, j \in \mathbb{N}\right\}$. Clearly $\left|x_{k_{i} l_{j}}-L\right|=\left|y_{k_{i}} l_{j}-L\right| \rightarrow 0$ as $i, j \rightarrow \infty$

(iii) $\Rightarrow$ (iv): Let $\left(x_{k l}\right)$ be a double sequence and there exists $M=\left\{\left(k_{i}, l_{j}\right): i, j \in \mathbb{N}\right\}$ such that $u_{2}(M)=1$ and $\lim _{i, j} x_{k_{i}} l_{j}=L$.

Now construct the sequences $\left(y_{k l}\right)$ and $\left(z_{k l}\right)$ as follows:

$$
y_{k l}=\left\{\begin{array}{ccc}
x_{k l} & ; & (k, l) \in M \\
L & ; & \text { otherwise }
\end{array},\right.
$$

and

$$
z_{k l}=\left\{\begin{array}{ccc}
0 & ; & (k, l) \epsilon M \\
x_{k l}-L & ; & \text { otherwise }
\end{array} .\right.
$$

From the above construction, it is obvious that $\left(y_{k l}\right)$ is convergent to $L$ and $s t_{u}^{2}-\lim _{k, l} z_{k l}=0$ and $x_{k l}=y_{k l}+z_{k l}$ for all $k, l \in \mathbb{N}$

(iv) $\Rightarrow$ (i): Suppose that there exists sequences such that $x_{k l}=y_{k l}+z_{k l}$ for all $k, l \in \mathbb{N} ;\left(y_{k l}\right)$ converges to $L$ and $s t_{u}^{2}-\lim _{k, l} z_{k l}=0$. For any $\varepsilon>0$, let $A=\left\{(k, l):\left|y_{k l}-L\right|<\frac{\varepsilon}{2}\right\}$ and $B=\left\{(k, l):\left|z_{k l}\right|<\frac{\varepsilon}{2}\right\}$. Then clearly $u_{2}(A)=u_{2}(B)=1$. This implies that

$$
u_{2}(A \cap B)=1, u_{2}\left(\left\{(k, l):\left|x_{k l}-L\right|<\varepsilon\right\}\right) \geq u_{2}(A \cap B) .
$$


By $u_{2}\left(\left\{(k, l):\left|x_{k l}-L\right|<\varepsilon\right\}\right)=1$, the sequence $\left(x_{k l}\right)$ is uniformly statistically convergent to $L$.

\section{A NEW SEQUENCE SPACE DEFINED BY A MODULUS FUNCTION}

In the section we define a new sequence space by means of a modulus function and we relate it to uniform statistical convergence.

Recall that a modulus [6] is a function from $[0, \infty)$ to $[0, \infty)$ such that

(i) $f(x)=0$ if and only if $x=0$,

(ii) $f(x+y) \leq f(x)+f(y)$ for $x, y \geq 0$,

(iii) $f$ is increasing,

(iv) $f$ is continuous at the right at 0 .

Because of (ii) $|f(x)-f(y)| \leq f(x-y)$ so that in view of (iv) $f$ is continuous on $[0, \infty)$. A modulus may be bounded or unbounded. For example $f(x)=x^{p}(0<p \leq 1)$ is unbounded and $f(x)=\frac{x}{x+1}$ is bounded.

The next sequence space of double sequences is motivated from [12] where in its single version is studied:

$$
=\left\{x=\left(x_{i j}\right): \lim _{p, q} \frac{1}{p q} \sum_{i, j=n+1, m+1}^{n+p, m+q} f\left(\left|x_{i j}-L\right|\right)=0 \text {, uniformly in } n \text { and } m\right\}
$$

Let us give the following two theorems.

Theorem 3. Let $f$ be any modulus. Then $\left[a c^{2}(f)\right] \subset s t_{u}^{2}$.

Proof. Suppose $x \epsilon\left[a c^{2}(f)\right]$ and $\varepsilon>0$. Then we have for every $n, m$

$$
\begin{gathered}
\frac{1}{p q} \sum_{i, j=n+1, m+1}^{n+p, m+q} f\left(\left|x_{i j}-L\right|\right) \geq \frac{1}{p q} \sum_{\substack{i, j=n+1, m+1 \\
\left|x_{i j}-L\right| \geq \varepsilon}}^{n+p, m+q} f\left(\left|x_{i j}-L\right|\right) \\
\geq f(\varepsilon) \frac{1}{p q}\left|E_{\varepsilon}\right|
\end{gathered}
$$

where

$E_{\varepsilon}=\left\{(i, j): n+1 \leq i \leq n+p, m+1 \leq j \leq m+q\right.$ and $\left.\left|x_{i j}-L\right| \geq \varepsilon\right\}$. This inequality implies that $x \epsilon s t_{u}^{2}$.

Theorem 4. $s t_{u}^{2}=\left[a c^{2}(f)\right]$ if and only if $f$ is bounded.

Proof. Suppose that $f$ is bounded and $x \epsilon s t_{u}^{2}$. Since $f$ is bounded there exists an integer $K$ such that $f\left(x_{i j}\right)<K$ for all $x_{i j} \geq 0, i, j=1,2, \ldots$. Then for each $n, m$ 
we have

$$
\begin{aligned}
\frac{1}{p q} \sum_{i, j=n+1, m+1}^{n+p, m+q} f\left(\left|x_{i j}-L\right|\right) & \\
= & \frac{1}{p q} \sum_{\substack{i, j=n+1, m+1 \\
\left|x_{i j}-L\right| \geq \varepsilon}}^{n+p, m+q} f\left(\left|x_{i j}-L\right|\right) \\
& +\frac{1}{p q} \sum_{\substack{i, j=n+1, m+1 \\
\left|x_{i j}-L\right|<\varepsilon}}^{n+p, m+q} f\left(\left|x_{i j}-L\right|\right) \\
\leq & \frac{1}{p q} K \max _{m, n \geq 0}\left|E_{\varepsilon}\right|+f(\varepsilon)
\end{aligned}
$$

Conversely suppose that $f$ is unbounded so that there is a positive sequence $\left(u_{i j}\right)$ such that $f\left(u_{i j}\right)=i^{2} j^{2}$ for $i, j=1,2, \ldots$

Now consider the sequence $x=\left(x_{i j}\right)$ defined by $x_{i j}=\left\{\begin{array}{cc}u_{i j} ; & i, j \text { square } \\ 0 ; & \text { otherwise }\end{array}\right.$.

Then we have

$$
\frac{1}{p q} \max _{m, n \geq 0}\left|\left\{n+1 \leq i \leq n+p, m+1 \leq j \leq m+q:\left|x_{i j}-L\right| \geq \varepsilon\right\}\right| \leq \frac{\sqrt{p} \sqrt{q}}{p q}
$$

Hence $s t_{u}^{2}-\lim x=L$. But $x \notin\left[a c^{2}(f)\right]$. Contradicting $s t_{u}^{2}=\left[a c^{2}(f)\right]$. This completes the proof.

As a result of Theorem 1 and Theorem 4 we easily obtain the next corollary.

Corollary 3. For bounded double sequences we have $\left[a c^{2}(f)\right]=u w_{p}^{2},(0<p<$ $\infty)$, if and only if $f$ is bounded.

The boundedness of the sequence can not be omitted. The next example demonstrates this fact.

Define $x=\left(x_{i j}\right)$ by

$$
x_{i j}=\left\{\begin{array}{rcc}
i j & ; & i \text { and } j \text { square } \\
0 & ; & \text { otherwise }
\end{array}\right.
$$

and take

$$
f(x)=\frac{x}{x+1}
$$

It is clear that $x$ is unbounded.

$$
\frac{1}{k l} \sum_{\substack{i, j=n+1, m+1 \\ i, j \text { square }}}^{n+k, m+l} f\left(\left|x_{i j}\right|\right) \leq \frac{1}{k l} \sum_{\substack{i, j=n+1, m+1 \\ i, j \text { square }}}^{n+k, m+l} 1
$$


$\leq \frac{1}{k l} \max _{m, n \geq 0} \mid\{n+1 \leq i \leq n+k, m+1 \leq j \leq m+l: i, j$ square $\} \mid$

So $x=\left(x_{i j}\right) \epsilon\left[a c^{2}(f)\right]$.

$$
\begin{gathered}
\frac{1}{k l} \sum_{i, j=n+1, m+1}^{n+k, m+l}\left|x_{i j}-0\right|^{p}=\frac{1}{k l} \sum_{i, j=n+1, m+1}^{n+k, m+l} x_{i j} p=\frac{1}{k l} \sum_{\substack{i, j=n+1, m+1 \\
i, j \text { square }}}^{n+k, m+l}(i j)^{p} \\
=\frac{1}{k l} \sum_{\substack{i, j=n+1, m+1 \\
i, j \text { square }}}^{n+k, m+l}(i j)^{p} \quad(*)
\end{gathered}
$$

One can find $p$ so that $(*)$ does not converge.

\section{REFERENCES}

[1] V. Baláž and T. Šalát, "Uniform density $u$ and corresponding $I_{u}$-convergence," Math. Commun., vol. 11, no. 1, pp. 1-7, 2006.

[2] T. C. Brown and A. R. Freedman, "Arithmetic progressions in lacunary sets," Rocky Mt. J. Math., vol. 17, pp. 587-596, 1987.

[3] T. C. Brown and A. R. Freedman, "The uniform density of sets of integers and Fermat's Last Theorem,” C. R. Math. Acad. Sci., Soc. R. Can., vol. 12, no. 1, pp. 1-6, 1990.

[4] R. Giuliano Antonini and G. Grekos, "Weighted uniform densities," J. Théor. Nombres Bordx., vol. 19, no. 1, pp. 191-204, 2007.

[5] P. Kostyrko, T. Šalát, and W. Wilczyński, “l-convergence," Real Anal. Exch., vol. 26, no. 2, pp. 669-685, 2001.

[6] I. J. Maddox, "Sequence spaces defined by a modulus," Math. Proc. Camb. Philos. Soc., vol. 100, pp. 161-166, 1986.

[7] H. I. Miller and C. Orhan, "On almost convergent and statistically convergent subsequences," Acta Math. Hung., vol. 93, no. 1-2, pp. 135-151, 2001.

[8] F. Móricz and B. E. Rhoades, "Almost convergence of double sequences and strong regularity of summability matrices," Math. Proc. Camb. Philos. Soc., vol. 104, no. 2, pp. 283-294, 1988.

[9] F. Móricz and B. E. Rhoades, "Some characterizations of almost convergence for single and double sequences," Publ. Inst. Math., Nouv. Sér., vol. 48(62), pp. 61-68, 1990.

[10] F. Móricz, "Statistical convergence of multiple sequences," Arch. Math., vol. 81, no. 1, pp. 82-89, 2003.

[11] M. Mursaleen and O. H. H. Edely, "Statistical convergence of double sequences," J. Math. Anal. Appl., vol. 288, no. 1, pp. 223-231, 2003.

[12] S. Pehlivan, "Strongly almost convergent sequences defined by a modulus and uniformly statistical convergence," Soochow J. Math., vol. 20, no. 2, pp. 205-211, 1994.

[13] A. Pringsheim, "On the theory of doubly infinite sequences of numbers. (Zur Theorie der zweifach unendlichen Zahlenfolgen.)," Math. Ann., vol. 53, pp. 289-321, 1900.

[14] B. Tripathy and B. C. Tripathy, "On I-convergent double sequences," Soochow J. Math., vol. 31, no. 4, pp. 549-560, 2005.

[15] B. C. Tripathy, "Statistically convergent double sequences," Tamkang J. Math., vol. 34, no. 3, pp. 231-237, 2003.

[16] F. Čunjalo, “Almost convergence of double subsequences," Filomat, vol. 22, no. 2, pp. 87-93, 2008. 
Authors' addresses

E. Tas

ANKARA UNIVERSITY FACULTY OF SCIENCE DEPARTMENT OF MATHEMATICS, TANDOĞAN 06100, ANKARA, TURKEY

E-mail address: emretas86@hotmail.com

T. Yurdakadmin

Current address: ANKARA UNIVERSITY FACULTY OF SCIENCE DEPARTMENT OF MATHEMATICS, TANDOGAN 06100, ANKARA, TURKEY

E-mail address: tugba-yurdakadimehotmail.com 